\title{
Molecular Dynamics Simulations To Determine the Effect of Supercritical Carbon Dioxide on the Structural Integrity of Hen Egg White Lysozyme
}

\author{
Hsuan-Liang Liu, ${ }^{, \dagger}$ Wei-Chan Hsieh, ${ }^{\dagger}$ and Hwai-Shen Liu ${ }^{\ddagger}$
}

Department of Chemical Engineering and Graduate Institute of Biotechnology, National Taipei University of Technology, No. 1 Sec. 3 Chung-Hsiao E. Rd., Taipei 10643, Taiwan, and Department of Chemical Engineering, National Taiwan University, No. 1 Sec. 4 Roosevelt Rd., Taipei 106, Taiwan

\begin{abstract}
In this study, various molecular dynamics simulations were conducted to investigate the effect of supercritical carbon dioxide on the structural integrity of hen egg white Iysozyme. The analyses of backbone root-mean-square deviation, radius of gyration, and secondary structure stability all show that supercritical $\mathrm{CO}_{2}$ exhibits the ability to increase the stability of this protein, probably as a result of the solvent with less polarity, where hydrophobic interactions stabilizing the native structure are weakened and simultaneously the local hydrogen bonds are strengthened, resulting in stabilization of the secondary structures. The hydrophobic cores in the $\alpha$ - and $\beta$-domains also play an important role in preventing this protein from thermal unfolding. As supercritical $\mathrm{CO}_{2}$ has been attractive for biomedical applications because of the advantages of mild critical condition, nonflammability, nontoxity, and the purity of the resulting products, the structural stabilizing effect found in this study strongly suggests that it is possible to increase the thermostability of hen egg white lysozyme by pretreatment with supercritical $\mathrm{CO}_{2}$, leading to better industrial applications of this protein.
\end{abstract}

\section{Introduction}

Fluids in the approximate temperature and pressure ranges $T \geq T_{c}$ and $P \geq P_{c}$ (where $c$ denotes the critical point) are known as supercritical fluids. They are highly compressible, and thus their solvent characteristics can be adjusted continuously from gaslike to liquidlike state with small change in pressure $(1,2)$. Supercritical fluids exhibit both liquidlike solvent properties and gaslike transport characteristics, which makes them useful for reactions and separations (1, 2). Carbon di oxide has been widely used as a solvent in supercritical fluid extraction (SFE) processes and has been attractive for biomedical applications (3) because of the advantages of mild critical condition (e.g., $\mathrm{T}_{\mathrm{c}}=31.1^{\circ} \mathrm{C}$ and $\mathrm{P}_{\mathrm{c}}=73.8 \mathrm{bar}$ ), nonflammability, nontoxity, and the purity of the resulting products.

Previous works by $\mathrm{Yeo}$ et al. $(4,5)$ have used a novel supercritical fluid antisolvent technique (SAS) to form insulin particles, and the subsequent secondary structure analysis by Raman spectroscopy revealed substantial loss of $\alpha$-helicity and marked increase in $\beta$-sheet and $\beta$-reverse turn content. The magnitude of structural distortion was found to be comparable to that of irreversibly denatured fibrils obtained by heating an aqueous insulin solution at $100{ }^{\circ} \mathrm{C}$ for $30 \mathrm{~min}$. Interestingly, despite the major conformational changes induced during SAS process, the insulin precipitates recovered their full biologi-

* To whom correspondence should be addressed. Phone: $+886-$ 2-2771-2171 ext. 2542. Fax: +886-2-2731-7117. E-mail: f10894@ ntut.edu.tw.

$\dagger$ National Taipei University of Technology.

₹ National Taiwan University. cal activity and native structure toward redissolution in aqueous solution (5). The SAS technique exposes proteins to organic and supercritical nonaqueous solvent, high pressure, and shearing forces. Any of these factors may potentially induce conformational changes of proteins. Winters et al. (6) have successfully shown that the applications of SAS technique to other proteins such as lysozyme and trypsin in supercritical $\mathrm{CO}_{2}\left(\mathrm{sCO}_{2}\right)$ exhibit behavior similar to that of insulin, that is, the biological activity can be totally recovered after redissolution in water. However, the structural perturbations induced during the SAS process were found to be protein-specific.

Hen egg white lysozyme (HEWL), constituting 3-4\% of the total hen protein content (7), was the third protein and the first enzyme to be determined by X-ray crystallography (8-10). It is composed of two domains (i.e., $\alpha$ and $\beta$-domains) with the active site cleft situated between them (Figure 1 ). The large $\alpha$-domain, composed of residues $1-39$ and $89-129$, has four $\alpha$-helices $(\alpha 1-\alpha 4)$ and a C-terminal $3_{10}$ helix, whereas the primary component of the smaller $\beta$-domain, composed of residues $40-$ 88 , is a three-stranded antiparallel $\beta$-sheet $(\beta 1-\beta 3)$, followed by a $3_{10}$ helix and an irregular loop containing two disulfide bridges The folding pathway of HEWL has been extensively studied (11). Early refolding experiments of HEWL suggested that the acquisition of secondary structure preceded the formation of tertiary structure $(12-14)$. The formation of secondary structure has been further confirmed by stop-flow CD studies $(15,16)$. These experimental results show that HEWL undergoes multiple folding pathways, that on the dominant folding pathway the $\alpha$-domain becomes substantially folded prior to the $\beta$-domain and that a significant minority of 


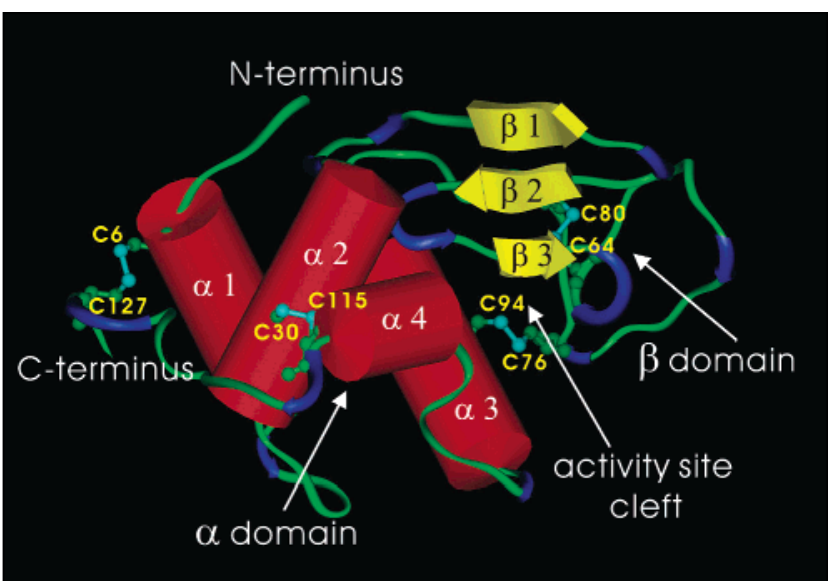

Figure 1. X-ray crystallographic structure of HEWL (50) visualized by Insight II program. The $\mathrm{N}$ - and $\mathrm{C}$-termini are indicated. $\alpha$-Helices $1-4(\alpha 1-\alpha 4)$ shown as red cylinders are numbered in sequential order from the $\mathrm{N}$ - to the $\mathrm{C}$-terminus. $\beta$-Strands $1-3(\beta 1-\beta 3)$ are shown as yellow arrows pointing from the $\mathrm{N}$ - to the $\mathrm{C}$-terminus. The locations of $\alpha$-domain (composed of residues 1-39 and 89-129), $\beta$-domain (composed of residues $40-88$ ), and the catalytic cleft between these two domains are indicated. The polypeptide backbones bel onging to the turn and random coil regions are shown in blue and green, respectively. The eight Cys residues forming the four disulfide linkages are labeled.

molecules follow a much faster route on which the domains fold at similar rates $(11,16,17)$. In addition, various molecular modeling protocols have also been conducted on HEWL and its complex with hexasaccharide to investigate the dynamics behaviors of this protein (18-21), including one of the pioneering applications of the temperature jump technique (22).

Several protein structures from extremophiles have provided clues as to how proteins can adapt to extreme temperatures or high salt and therefore helped unravel details of the physical constraints governing the structure and evolution of proteins (23-25). Temperature usually causes simultaneous changes in volume and thermal energy of a protein, whereas pressure causes changes only in volume (26). Therefore, pressure effects are of interest because protein compressibility is directly related to the structural and conformational fluctuations of proteins at normal atmospheric pressure $(27,28)$. To investigate the characteristics of HEWL under extreme conditions, denaturation of HEWL at high pressure has been studied by high resolution ${ }^{1} \mathrm{H}$ NMR spectroscopy $(29,30)$. In addition to sperm whale myoglobin (31), the crystal structure of HEWL at a hydrostatic pressure of 1,000 atm has been determined by X-ray diffraction to a nominal resolution of $2 \AA$, indicating that the contraction of this enzyme was nonuniformly distributed under high pressure (32). Recently, pressure-dependent changes in the solution structure of HEWL has been investigated, showing that the $\alpha$-domain is compressed by approximately $1 \%$ and the interdomain region is also compressed, whereas the $\beta$-domain displays very little overall compression (33).

In addition to the pressure effect on the structural changes of proteins, solvents also play an important role in determining the conformation of proteins. In general, secondary structures stabilized in solvents with low polarity are considered to be the initiation site of protein folding $(34,35)$. For example, addition of alcohols successfully converts melittin from unfolded state into a monomeric $\alpha$-helical structure $(36,37)$. The effects of alcohols can be attributed to some extent by the de creased polarity of the solvent (38-40). In solvents of low polarity, hydrophobic interactions stabilizing the native structure or the protein aggregate are weakened, and simultaneously the local hydrogen bonds are strengthened, resulting in denaturation or dissolution and stabilization of the extended $\alpha$-helical structures.

Usually, high temperature, mechanical stresses, and pressure are the main causes enhancing enzyme inactivation in industrial systems. However, HEWL has been shown to exhibit higher thermal stability by pretreatment with $\mathrm{sCO}_{2}$ for $1 \mathrm{~h}$ (unpublished data). The effect of the $\mathrm{SCO}_{2}$ on enhancing the thermal stability of HEWL is likely to be attributed to either the pressure effect or the solvent characteristics of this fluid. Molecular dynamics (MD) simulations have provided a powerful tool to understand the dynamics of a protein at atomic detail, which can lead to significant insights into the atomic motions and the machinery underlying the protein function (41-43). Previously, we have successfully conducted several MD simulations to investigate the conformational changes of various proteins under different conditions (44-49). To investigate the effect of $\mathrm{sCO}_{2}$ on the structural integrity of $\mathrm{HEWL}$, two sets of experiments were performed in this study: (1) HEWL was placed in a lattice full of $\mathrm{SCO}_{2}$, followed by energy minimization calculations and 200 ps MD simulations at its critical condition (i.e., $305 \mathrm{~K}$ and 73.8 bar). The resulting structure at 200 ps was then subjected to a lattice full of water molecules, followed by additional 200 ps MD simulations at 1 atm and 310, 450, and $600 \mathrm{~K}$. (2) HEWL was placed in a lattice full of water molecules, followed by energy minimization calculations and 400 ps MD simulations at 1 atm and 310, 450, and $600 \mathrm{~K}$ for comparison.

\section{Materials and Methods}

The initial X-ray crystal lographic structure of HEWL (EC 3.2.1.17) solved at $1.6 \AA$ (Figure 1 ) was taken from the Protein Data Bank (PDB entry 1LKS) (50). The energy minimizations and MD simulations of HEWL were conducted by Insight II program (Accelyrs, San Diego, CA) with the force field Discover CVFF (consistent valence force field) (51-53) in the SGI 0200 workstation with 64-bit HIPS RISC R12000 $2 \times 270 \mathrm{MHz}$ CPU and PMC-Sierra RM 7000A 350 MHz processor (Silicon Graphics, I nc., Mountain View, CA). The force field parameters in CVFF were devel oped by computing the properties of nearly 2000 different macromolecules such as proteins, nucleic acids, carbohydrates, and lipids, resulting in over $2,000,000$ quantum mechanically computed energies and energy derivatives. The X-ray crystallographic structure of HEWL (129 residues and 1,951 atoms in total) was subjected to energy minimization calculations by conjugate gradient method with 10,000 iterations to be used as starting lowest energy structure for future structure comparison.

The energy-minimized HEWL was placed in the center of two pseudo unit cells of the size $55 \times 55 \times 55 \AA^{3}$ and soaked with water and carbon dioxide molecules into the unit cell, respectively. To arrange the soaked solvent randomly, solvent molecular were submitted to 10,000 iterations by conjugate gradient method, keeping the atoms of HEWL fixed during the solvent randomization step. The range of cutoff radius was set as $10 \AA$ for both nonbonded electrostatic and van der Waals interactions. The minimum image (molecule migrates out of the solvent box will not be re-imaged at the end of MD simulation and minimization) periodic boundary conditions (PBC) were used to keep constant volume during 


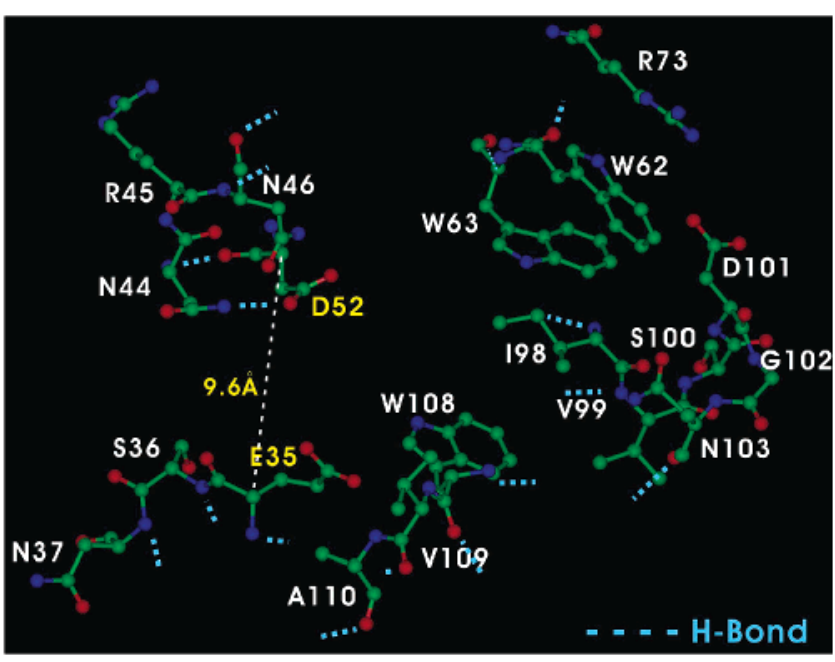

Figure 2. Geometry of the catalytic d eft located between the $\alpha$ - and $\beta$-domain (19). The distance, $9.6 \AA$, between the catalytic residues $\mathrm{E} 35$ and $\mathrm{D} 52$ is also shown.

the MD courses and decrease the total CPU time of MD simulation.

The energy-minimized $\mathrm{HEWL} / \mathrm{SCO}_{2}$ system was then submitted to 200 ps MD simulations at its critical condition (i.e., $305 \mathrm{~K}$ and 73.8 bar) after equilibrating for about 10 ps using the Discover module of the Insight II program. The resulting structure at 200 ps was then subjected to a lattice full of water molecules, followed by additional 200 ps MD simulations at 1 atm and 310, 450, and $600 \mathrm{~K}$. F or comparison, the energy-minimized HEWL/ water system was submitted to 400 ps MD simulations at 1 atm and 310,450 , and $600 \mathrm{~K}$. The temperature and pressure were maintained constant for each MD simulation according to the method described by Berendsen et al. (54). The time-step of the MD simulations was $1 \mathrm{fs}$. The trajectories and coordinates of HEWL were saved every 2 ps for further analysis.

For each MD simulation, the backbone $\mathrm{C}_{\alpha}$ root-meansquare deviation (RMSD), radius of gyration and accessible surface area (ASA) of the entire HEWL, the $\alpha$ - and $\beta$-domains, the catalytic cleft (19) (Figure 2), and the hydrophobic cluster in the $\alpha$-domain (55) (F igure 3 ) were recorded during the entire course of each MD simulation with reference to the starting structure. The RMSDs were calculated after optimal superimposition of the coordinates to remove translational and rotational motion (56). The secondary structure was predicted according to DSSP (57), in which pattern recognition of hydrogen-bonding was correlated to the geometrical features. The default hydrogen bonding energy criterion of $-0.5 \mathrm{kcal} / \mathrm{mol}$ was used. The helicity, $\mathrm{H}(\mathrm{t})$, and the residual $\beta$-strand content, $\mathrm{B}(\mathrm{t})$, were defined as the ratio of the number of the residual $\mathrm{H}$ bonds at time to the number of the total $\mathrm{H}$ bonds in each $\alpha$-helix and $\beta$-strand of the starting structure, respectively. The averaged helicity, $\langle\mathrm{H}\rangle$, and the averaged $\beta$-strand content, $\langle\mathrm{B}\rangle$, were then defined by $1 / t_{f} \int_{0}^{t_{f}} H(t) d t$ and $1 / t_{f} \int_{0}^{t_{f}} B(t) d t$, where $t_{f}$ is the total length of each MD simulation, respectively.

\section{Results and Discussion}

HEWL has 129 amino acid residues and consists of two domains separated by a catalytic cleft in which hexasaccharides are bound (Figure 1). Overall, it consists of $34 \% \alpha$-helix, $15 \% \beta$-sheet, $9 \% 3_{10}$ helix, and $42 \%$ random coils and turns. In the $\alpha$-domain $\alpha$-helices 1,3 , and 4 are relatively exposed, whereas $\alpha$-helix 2 is more in the

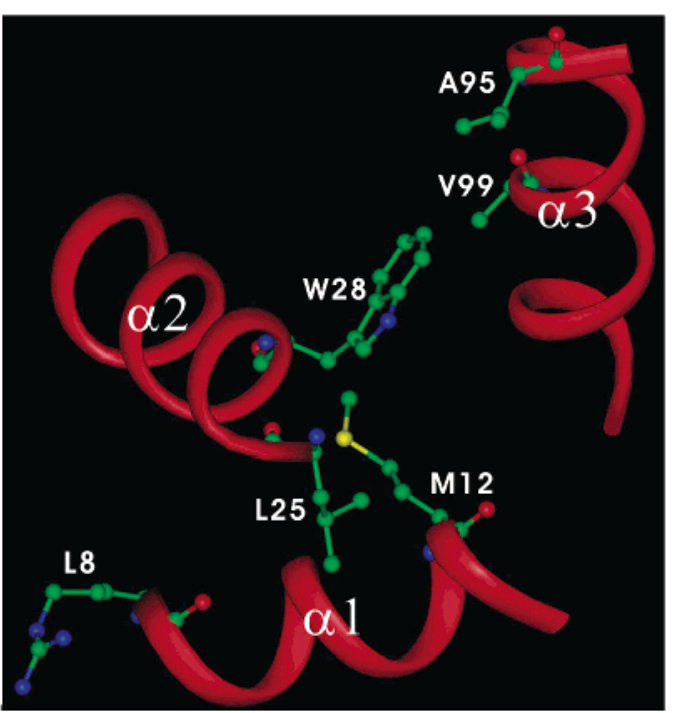

Figure 3. Illustration of the hydrophobic core located in the $\alpha$-domain (55).

interior with only the ends partly exposed to solvent. In the $\beta$-domain, $\beta$-strand 1 runs along the protein surface near the reducing end of the catalytic cleft, and the other two strands are in the protein interior. To investigate the effect of $\mathrm{SCO}_{2}$ on the structural integrity of $\mathrm{HEWL}$, various MD simulations were conducted in various solvent systems in the present study. As shown in Figure $4 \mathrm{~A}$, the backbone RMSD of the entire HEWL increased dramatically at $600 \mathrm{~K}$ when HEWL was placed in water during the entire 400 ps MD simulation, whereas it was considerably smaller at all simulations temperatures when $\mathrm{HEWL}$ was pretreated with $\mathrm{sCO}_{2}$ for 200 ps MD simulation and subsequently submitted to another 200 ps MD simulations in water at various temperatures. Similarly, the backbone RMSDs for the $\alpha$ - and $\beta$-domains, the catalytic cleft (19), and the hydrophobic area in the $\alpha$-domain (55) (Figure 4B-E) were all smaller when $\mathrm{HEWL}$ was pretreated with $\mathrm{SCO}_{2}$ compared to those in water at various temperatures. These results indicate that $\mathrm{sCO}_{2}$ exhibits the ability to reduce the structural variations at high temperatures, which in turn implies that the thermostability of HEWL could be enhanced when it is pretreated with $\mathrm{SCO}_{2}$.

Most of the high-pressure computer simulations re ported so far have been of the MD type (58). The first high-pressure MD simulations on BPT1 were reported by Kitchen et al. (59). No changes in the conformation were detected at 10 kbar; only the increased hydration of certain amino acids was observed. Subsequent MD simulations revealed that changes in the secondary structures between 10 and 15 kbar were observed (60). These changes could be correlated with changes in the secondary structure observed with high-pressure infrared studies (61). Furthermore, no net unfolding of Iysozyme was observed at $10 \mathrm{kbar}$ after 210 ps (20). However, fluorescence (62) as well as Raman (63) studies indicate that the protein unfolds at about 5 kbar. According to the above observations, one may ask the question whether the pressure-temperature behavior of protein is unique among various biomacromolecules. Previously, Ezaki and Hayashi (64) have shown that starch also forms a gel by the application of pressure. This suggests that proteins and starch show a similar behavior with regard to temperature and pressure, which raises the question on the presumed role of hydrophobic interactions in the stability of protein (58). 

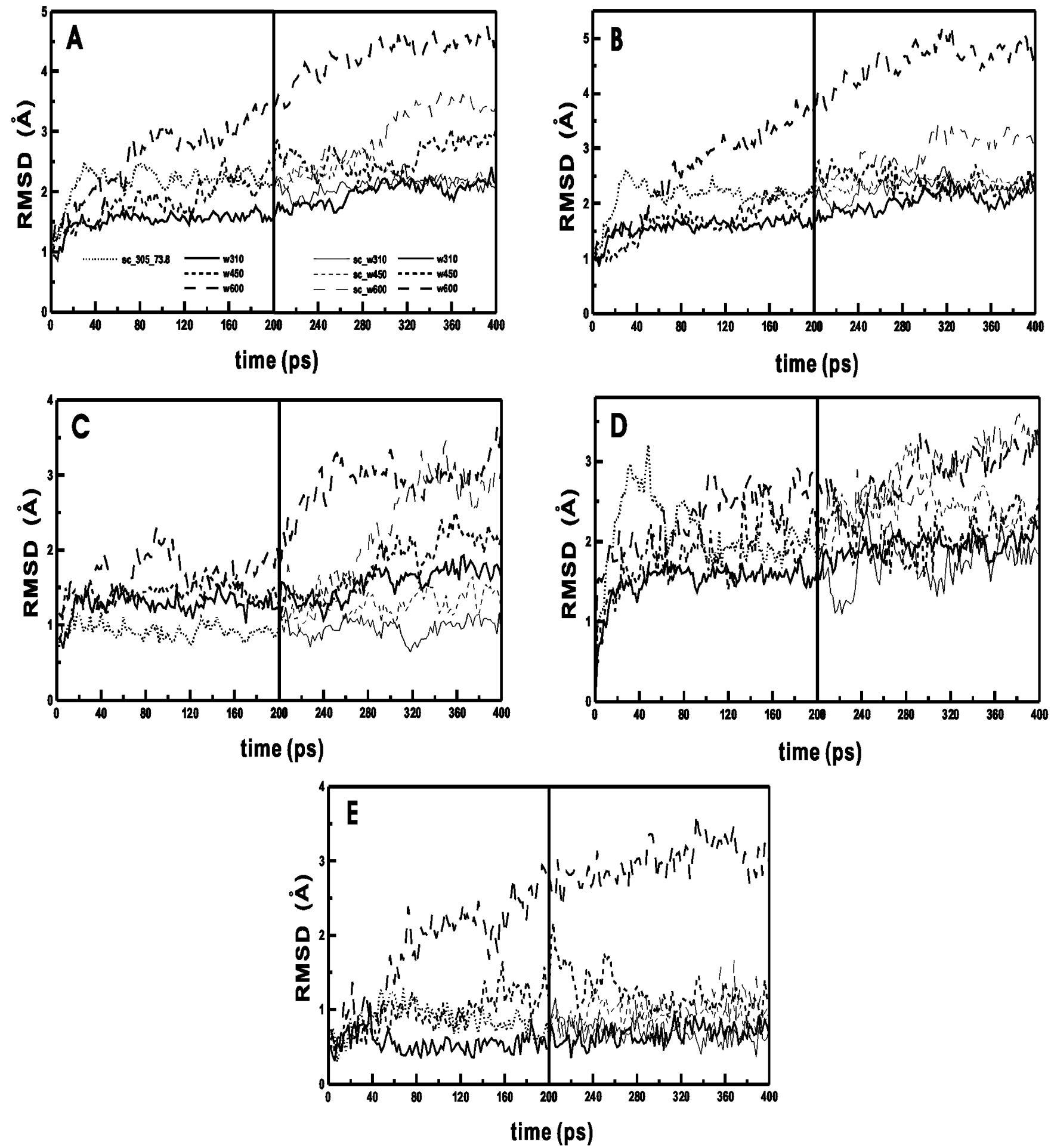

Figure 4. Backbone RMSD of (A) the entire HEWL, (B) the $\alpha$-domain, (C) the $\beta$-domain, (D) the catalytic deft, and (E) the hydrophobic core in the $\alpha$-domain. Abbreviations: sc 305_73.9, MD simulation carried out in $\mathrm{SCO}_{2}$ at its critical condition for 200 ps; w310, w450, and w600, MD simulations conducted in water at 310, 450, and $600 \mathrm{~K}$ for 400 ps, respectively; sc_w310, sc_w450, and sc_w600, MD simulation performed in $\mathrm{sCO}_{2}$ for $200 \mathrm{ps}$ and then in water for another $200 \mathrm{ps}$ at 310, 450, and $600 \mathrm{~K}$, respectively.

Previously, radius of gyration has been chosen to investigate the effect of pressure on protein compressibility $(58,65)$, where protein is compressed when the radius of gyration decreases (20). As shown in Figure 5, the values of radius of gyration of the entire HEWL or of various structural el ements were all more stable when the HEWL was pretreated with $\mathrm{SCO}_{2}$, particularly in the hydrophobic core of the $\alpha$-domain, which is formed by $\alpha$-helices 1,2 , and 3 (Figure 3 ). It indicates that this structural element is resistant to compressibility induced by high pressure. This result is consistent with the previous findings that the main effect of the pressure is a compaction of the hydrophobic core part of the protein consisting of a bulky side-chains (30), the $\alpha$-domain is compressed as a result of tighter packing between helices, and the $\beta$-domain displays very little overall compression but undergoes more structural distortion against high pressure (33). Previous X-ray diffraction study of Iysozyme crystals at a hydrostatic pressure of 1 kbar (32) has shown a differential compressibility between the two 

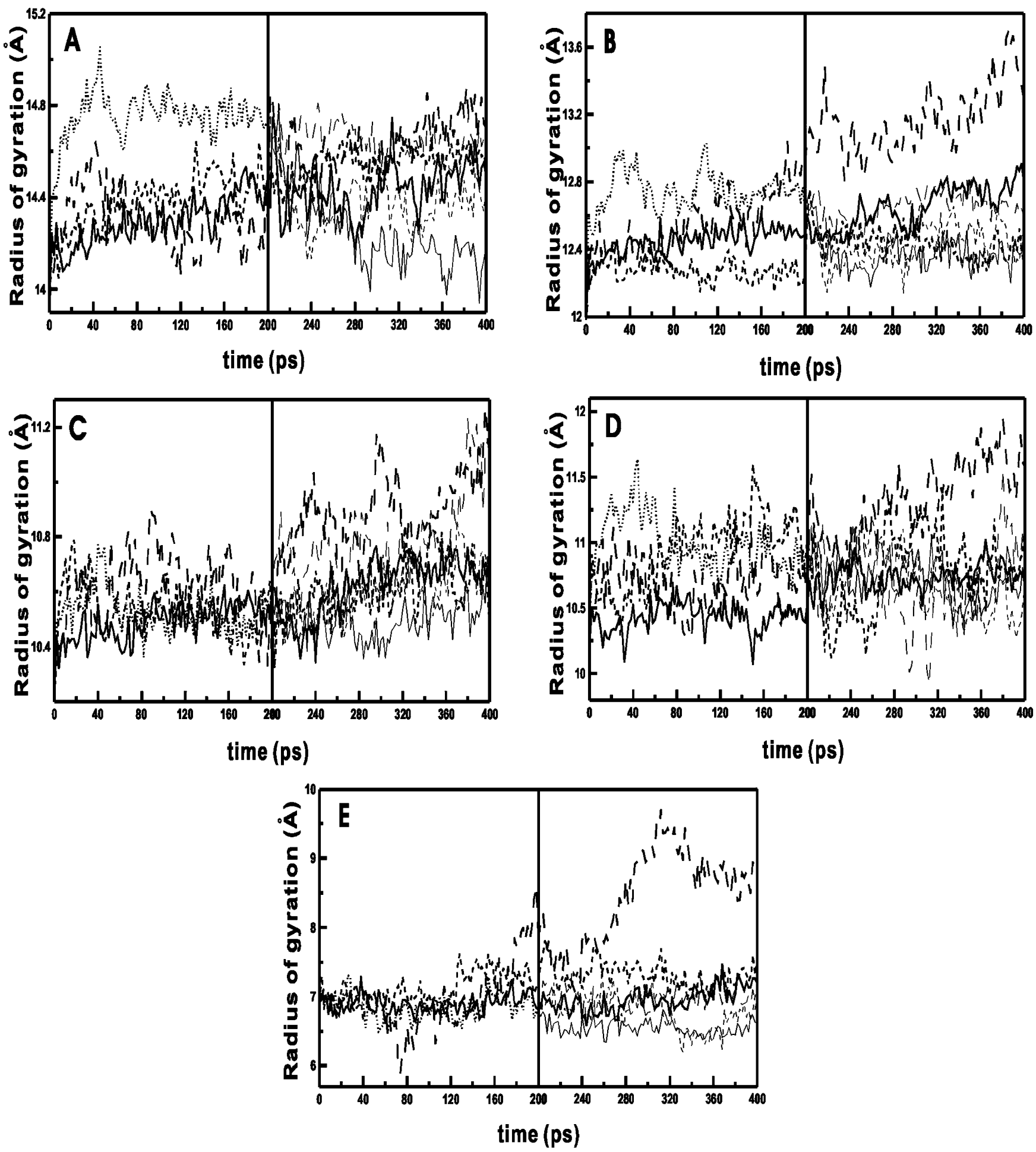

Figure 5. Radius of gyration of (A) the entire HEWL, (B) the $\alpha$-domain, (C) the $\beta$-domain, (D) the catalytic cleft, and (E) the hydrophobic core in the $\alpha$-domain. Abbreviations are the same as in Figure 4.

domains, the $\beta$-domain being essentially incompressible while the $\alpha$-domain and the interdomain catalytic cleft contracted under pressure. The present results show that $\mathrm{sCO}_{2}$ can protect $\mathrm{HEWL}$ from pressure denaturation, probably because of its less polar solvent properties, which is in good agreement with the previous statements that buried water molecules play an important role in conformational fluctuation at normal pressures and are implicated as the nucleation sites for structural changes leading to pressure denaturation or channel opening (33).
Temperature jump technique has been intensively applied to investigate the unfolding mechanism of various proteins $(44-46,66,67)$. Applied to lysozyme, this unfolding method resulted in an almost complete loss of secondary structure and the splitting of the hydrophobic core into four main clusters (20). The overall shape of the protein, however, remained relatively compact. The folding model supported by lysozyme MD simulation is a hydrophobic collapse prior to or concomitant with secondary and tertiary structure formation (68). To investigate the stability of the secondary structures (i.e., 
Table 1. Average Secondary Structure Content of Each $\alpha$-Helix and $\beta$-Strand during Various MD Simulations Conducted in This Study

\begin{tabular}{|c|c|c|c|c|c|c|c|c|}
\hline \multirow[b]{2}{*}{ secondary structure } & \multirow[b]{2}{*}{ residues } & \multicolumn{6}{|c|}{ av secondary structure content, $\langle\mathrm{H}\rangle$ or $\langle\mathrm{B}\rangle(\%)$} & \multirow[b]{2}{*}{$\mathrm{sCO}_{2} \mathrm{w}_{600} \mathrm{a}$} \\
\hline & & w310a & $w 450^{a}$ & W600a & $\mathrm{sCO} 2^{\mathrm{a}}$ & $\mathrm{sCO}_{2} \_\mathrm{w} 310^{\mathrm{a}}$ & $\mathrm{sCO}_{2} \mathrm{w}_{4} 40^{\mathrm{a}}$ & \\
\hline$\alpha$-helix 1 & $5-15$ & 90.6 & 81.9 & 0.5 & 82.2 & 85.2 & 75.1 & 75.4 \\
\hline$\alpha$-helix 2 & $25-36$ & 85.1 & 78.5 & 35.2 & 94.1 & 87.5 & 80.5 & 63.5 \\
\hline$\alpha$-helix 3 & $89-100$ & 91.5 & 92.8 & 26.9 & 69.2 & 87.8 & 84.1 & 78.8 \\
\hline$\alpha$-helix 4 & $109-112$ & 90.2 & 7.0 & 10.5 & 34.5 & 7.1 & 1.2 & 0 \\
\hline$\beta$-strand 1 & $43-45$ & 94.3 & 65.3 & 38.1 & 67.3 & 81.4 & 84.2 & 65.6 \\
\hline$\beta$-strand 2 & $51-53$ & 94.0 & 74.6 & 48.6 & 69.3 & 82.2 & 88.3 & 77.6 \\
\hline$\beta$-strand 3 & $58-59$ & 83.1 & 90.0 & 27.3 & 84.4 & 79.3 & 86.1 & 63.3 \\
\hline
\end{tabular}

a The conditions of these MD simulations are described in detail in Figure 4.

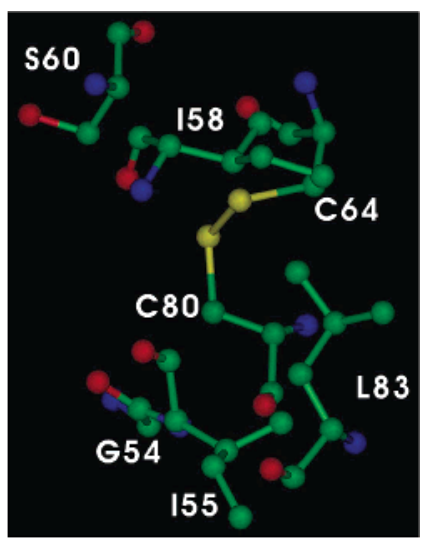

Figure 6. Presumed hydrophobic core located in the $\beta$-domain.

$\alpha$-helices in the $\alpha$-domain and $\beta$-strands in the $\beta$-domain) of $\mathrm{HEWL}$, the average helicity $\langle\mathrm{H}\rangle$ and the average $\beta$-strand content $\langle\mathrm{B}\rangle$ for each $\alpha$-helix and $\beta$-strand were calculated, respectively. As shown in Table 1 , both $\langle\mathrm{H}\rangle$ and $\langle B\rangle$ decreased with increasing temperature. Usually, both $\langle\mathrm{H}\rangle$ and $\langle\mathrm{B}\rangle$ were markedly higher when $\mathrm{HEWL}$ was pretreated with $\mathrm{SCO}_{2}$, except for $\alpha$-helix 4 . In water environment, the destruction of the secondary structure in the $\alpha$-domain followed the order of $4 \rightarrow 1 \rightarrow 2 \rightarrow 3$. $\alpha$-Helix 4 unfolded first probably because it is located on the surface of this domain. $\alpha$-Helix 1 unfolded from its $\mathrm{N}$-terminus because its $\mathrm{C}$-terminus is protected by the hydrophobic core through the interaction with $\mathrm{M} 12$, L25, and W28 (Figure 3). In contrast, $\alpha$-helices 2 and 3 were stabilized by the disulfide bridge located in the interior of this domain. In addition, $\alpha$-helix 3 unfolded from its C-terminus, which is consistent with the work by Kazmirshi and Daggett (55). The secondary structure content in the $\beta$-domain is less than that in the $\alpha$-domain, and thus the structural integrity of this domain may not be a result of the stability of the secondary structure in this domain. Whereas $\beta$-strand 1 is located outside this domain, $\beta$-strands 2 and 3 are located in the interior near the catalytic cleft (Figure 1). The disulfide bond formed by Cys64 and Cys80 is more or less responsible for maintaining the structural integrity of the $\beta$-domain during the simulations conducted in this study. F urthermore, as shown in Figure 6, five residues, Gly54, Ile55, Leu58, Ile60, and Leu83, form a hydrophobic core around this disulfide bond, which in turn helps to stabilize the structure of the $\beta$-domain.

Figure 7 shows the snapshots of HEWL during various MD simulations conducted in this study. The secondary structure propensity of HEWL was predicted according to DSSP (57) during the entire MD course, and the results are shown in Figure 8 . It is obviously that pretreatment of $\mathrm{HEWL}$ with $\mathrm{sCO}_{2}$ can stabilize the

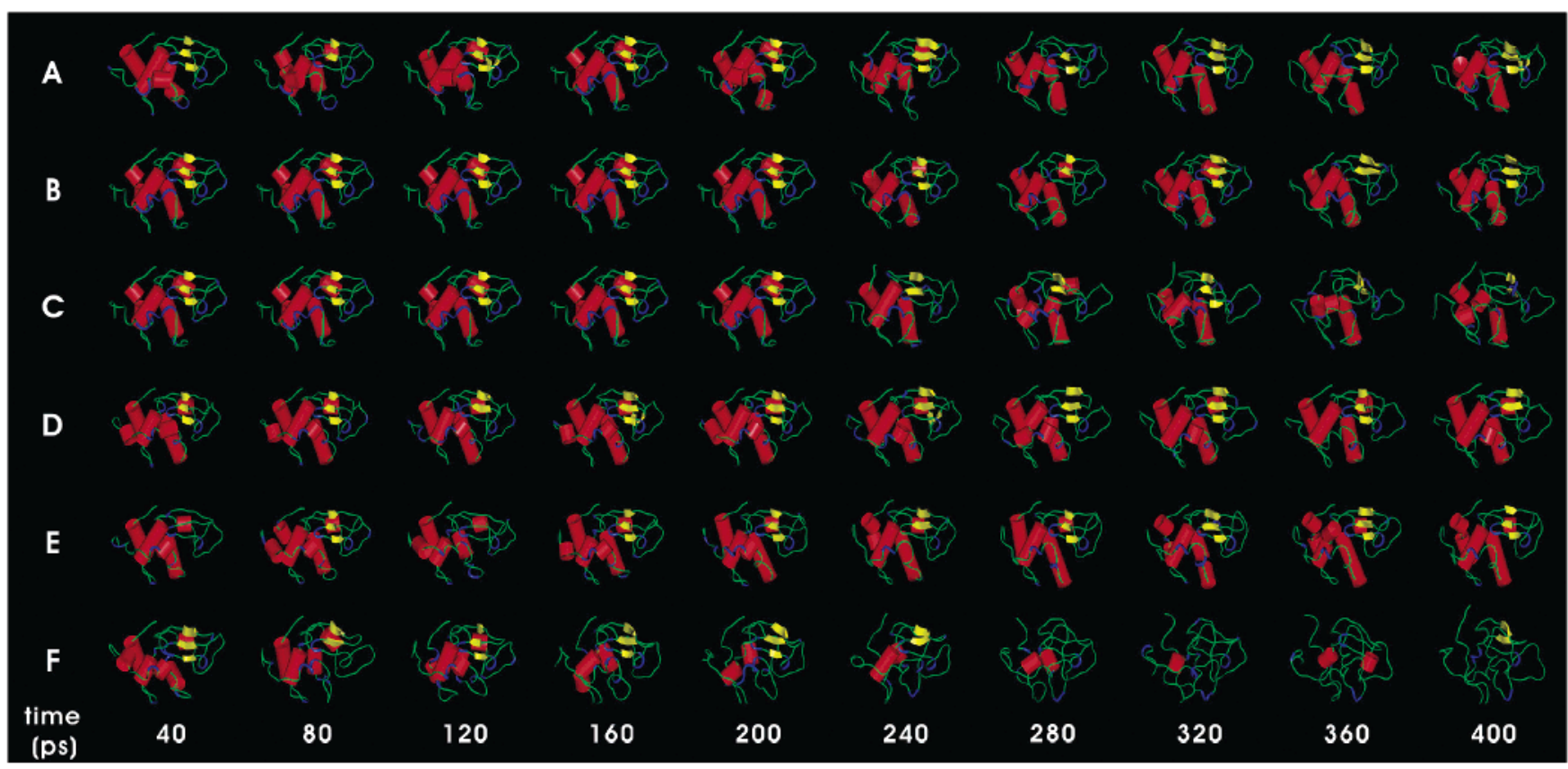

Figure 7. Snapshots of HEWL from various MD simulations: (A) 200 ps MD simulation in $\mathrm{SCO}_{2}$ and then 200 ps MD simulation in water at $310 \mathrm{~K}$, (B) 200 ps MD simulation in sCO 2 and then 200 ps MD simulation in water at $450 \mathrm{~K}$, (C) 200 ps MD simulation in $\mathrm{sCO}_{2}$ and then $200 \mathrm{ps}$ MD simulation in water at $600 \mathrm{~K}$, (D) 400 ps MD simulation in water at $310 \mathrm{~K}$, (E) 400 ps MD simulation in water at $450 \mathrm{~K}$, and $(\mathrm{F}) 400 \mathrm{ps}$ MD simulation in water at $600 \mathrm{~K}$. The secondary structures are illustrated the same as in Figure 1. 

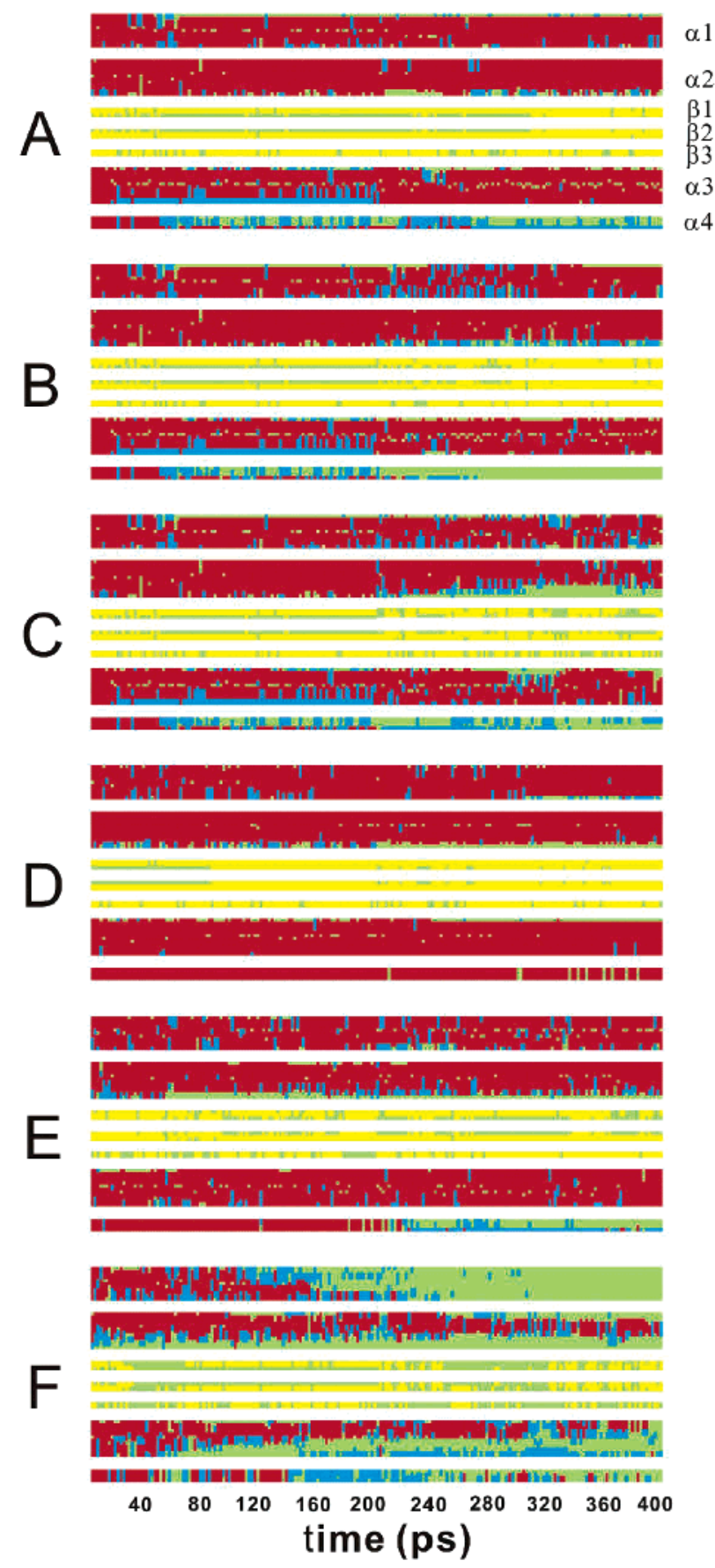

helix $\square$ sheet $\square$ turn $\square$ coil

Figure 8. Secondary structures as a function of MD simulation time. The conditions for each MD simulations are the same as in Figure 7. $\alpha$-Helix, $\beta$-sheet, turn, and coil estimated according to DSSP (57) are shown in red, yellow, blue, and green, respectively.

secondary structures to a great extent, particularly at $600 \mathrm{~K}$, as a result of the solvent effect. In solvent of lower polarity, hydrophobic interactions stabilizing the native structure are weakened, and simultaneously the local hydrogen bonds are strengthened, resulting in stabilization of the secondary structures. In other words, the weaker dielectric constant of $\mathrm{sCO}_{2}$ possibly reduces the hydrogen bonding between amide protons and surrounding solvent molecules and simultaneously promotes the intramolecular hydrogen bonding in the secondary structures and therefore stabilizes them. The effect of $\mathrm{sCO}_{2}$ on stabilizing the secondary structures of HEWL is most likely due to its ability to form clusters on the surface of HEWL effectively, favoring the formation of intramolecular hydrogen bonds instead of intermolecular hydrogen bonds and promoting the formation of stable secondary structures. The snapshots from various MD simulations also indicate that the $\alpha$ - and $\beta$-domains probably unfolded independently, which is consistent with the previous findings that the first step in unfolding of lysozyme is an "unlocking" of the two independent folding/unfolding domains (69), the reverse of the final step of folding (11). Furthermore, the present results, in which HEWL lost most of its secondary structure in water at $600 \mathrm{~K}$ at the end of $400 \mathrm{ps}$ MD simulation, are in good agreement with the findings by Mark and van Gunsteren (22), where unfol ding of HEWL proceeds via a metastable molten globulelike state, though with relatively little secondary structure.

In conclusion, the structural integrity of HEWL can be successfully enhanced by pretreatment with $\mathrm{SCO}_{2}$ by backbone RMSD, radius of gyration, and secondary structure analyses. The critical pressure of $\mathrm{SCO}_{2}$ is not high enough to cause the structural denaturation of $\mathrm{HEWL}$. The stabilizing effect of supercritical $\mathrm{CO}_{2}$ is likely due to redued pol arity of the solvent, where hydrophobic interactions stabilizing the native structure are weakened and simultaneously the local hydrogen bonds are strengthened, resulting in stabilization of the secondary structures. The hydrophobic cores in these two domains play an important role in preventing $\mathrm{HEWL}$ from thermal unfol ding. As $\mathrm{SCO}_{2}$ has been attractive for biomedical applications because of the advantages of mild critical condition, nonflammability, nontoxity, and the purity of the resulting products, the structural stabilizing effect found in this study suggests that it is possible to increase the thermostability of $\mathrm{HEWL}$ by pretreatment with $\mathrm{SCO}_{2}$ resulting in better industrial applications of this protein.

\section{Acknowledgment}

The authors thank the National Science Council of Taiwan for financial support (project NSC 92-2214-E027-009).

\section{References and Notes}

(1) J ohnston, K. P.; Penninger, J . M. L. Supercritical fluid science and technology. ACS symposium series 406; American Chemical Society: Washington, DC, 1989.

(2) Kiran, E.; Levelt, J . M. H. Supercritical Fluids. Fundamental for Application, NATO ASI Ser. E; Kluwer: Dordrecht, 1994; Vol. 273.

(3) Bruno, T. J.; Ely, J . F. Supercritical Fluid Technology: Reviews in Modern Theory and Applications; CRC: Boston, 1991.

(4) Yeo, S.-D.; Lim, G.-B.; Debenedetti, P. G.; Bernstein, H. Formation of microparticulate protein powders using a supercritical fluid antisolvent. Biotechnol. Bioeng. 1993, 41, 341-346.

(5) Yeo, S.-D.; Debenedetti, P. G.; Patro, S.; Przybycien, T. M. Secondary structure characterization of microparticulate insulin powders. J. Pharm. Sci. 1994, 83, 1651-1656.

(6) Winters, M. A.; Knutson, B. L.; Debenedetti, P. G.; Sparks, H. G.; Przybycien, T. M.; Stevenson, C. L.; Prestrelsk, S. J. Precipitation of proteins in supercritical carbon dioxide. J. Pharm. Sci. 1996, 85, 586-594.

(7) Proctor, V. A.; Cunningham, F. E. The chemistry of lysozyme and its use as a food preservative and a pharmaceutical. CRC Crit. Rev. Food Sci. Nutr. 1998, 26, 359-395.

(8) Blake, C. C. F.; Fenn, R. H.; North, A. C. T.; Phillips, D. C.; Polijak, R. J . Structure of lysozyme. Nature 1962, 196, 11731176.

(9) Dickerson, R. E.; Reddy, J . M.; Pinkerton, M.; Steinrauf, L. K. Structure of lysozyme: A Fourier map of the electron 
density at $6 \AA$ resolution obtained by X-ray diffraction. Nature 1962, 196, 1178-1181.

(10) Stanford, R. H.; Marsh, R. E.; Corey, R. B. Studies on the structure of lysozyme. I. A non-centrosymmetric projection to $4 \AA$ resolution. Nature 1962, 196, 8-13.

(11) Dobson, C. M.; Evans, P. E.; Radford, S. E. Understanding how proteins fold: the lysozyme story so far. Trends Bi ochem. Sci. 1994, 19, 31-37.

(12) I keguchi, M.; Kuwajima, K.; Mitani, M.; Sugai, S. Evidence for identity between the equilibrium unfolding intermediate and a transient folding intermediate: a comparative study of the folding reactions of $\alpha$-lactalbumin and Iysozyme. Biochemistry 1986, 25, 6965-6972.

(13) Kato, S.; Okamura, M.; Shimanoto, N.; Utiyama, H. Spectral evidence for a rapidly formed stractural intermediate in the refolding kinetics of hen egg-white lysozyme. Biochemistry 1981, 20, 1080-1085.

(14) Kuwajima, K.; Hiraoka, Y.; I keguchi, M.; Sugai, S. Comparison of the transient folding intermediates in lysozyme and $\alpha$-lactalbumin. Biochemistry 1985, 24, 874-881.

(15) Chaffotte, A. F.; Guillou, Y.; Goldberg, M. E. Kinetic resolution of peptide bond and side chain far-UV circular dichroism during the folding of hen egg white Iysozyme. Biochemistry 1992, 31, 9694-9702.

(16) Radford, S. E.; Dobson, C. M.; Evans, P. A. The folding of hen lysozyme involves partially structured intermediates and multiple pathways. Nature 1992, 358, 302-307.

(17) Keifhaber, T. Kinetic traps in lysozyme fol ding. Proc. Natl. Acad. Sci. U.S.A. 1995, 92, 9029-9033.

(18) Post, C. B.; Brooks, B. R.; Karplus, M.; Dobson, C. M.; Artymiuk, P. J .; Cheetham, J . C.; Philips, D. C. Molecular dynamics simulations of native and substrate-bound lysozyme. J. Mol. Biol. 1986, 190, 455-479.

(19) Post, C. B.; Dobson, C. M.; Karplus, M. On the evaluation of interproton distances for three-dimensional structure de termination by NMR using a relaxation rate matrix analysis. Proteins: Struct., Funct., Genet. 1989, 5, 337-354.

(20) Hünenberger, P. H.; Mark, A. E.; van Gunsteren, W. F. Computational approaches to study protein unfolding: hen egg white lysozyme as a case study. Proteins 1995, 21, 196213.

(21) Smith, L. J .; Mark, A. E.; Dobson, C. M.; van Gunsteren, W. F. Comparison of MD simulations and NMR experiments for hen lysozyme: Analysis of local fluctuations, cooperative motions and global changes. Biochemistry 1995, 34, 1091810931.

(22) Mark, A. E.; van Gunsteren, W. F. Simulation of the thermal denaturation of HEW-Iysozyme: Trapping the molten gl obule state. Biochemistry 1992, 31, 7745-7748.

(23) Leiros, H. K. S.; Willassen, N. P.; Smalås, A. O. Structural comparison of psychrophilic and mesophilic trypsins. Elucidating the molecular basis of cold-adaptation. Eur. J. Biochem. 2000, 267, 1039-1049.

(24) Mevarech, M.; Frolow, F.; Gloss, L. M. Halophilic enzymes: proteins with a grain of salt. Biophys. Chem. 2000, 86, 155-164.

(25) Szilágyi, A.; Závodszky, P. Structural differences between mesophilic, moderately thermophilic and extremely thermophilic protein subunits: results of a comprehensive survey. Struct. Fold. Des. 2000, 8, 493-504.

(26) Paci, E.; Marchi, M. Intrinsic compressibility and volume compression in solvated proteins by molecular dynamics simulation. Proc. Natl. Acad. Sci. U.S.A. 1996, 93, 1160911614.

(27) Cooper, A. Thermodynamic fluctuations in protein molecules. Proc. Natl. Acad. Sci. U.S.A. 1976, 73, 2740-2741.

(28) Gekko, K.; Hasegawa, Y. Compressibility-structure relationship of globular proteins. Biochemistry 1986, 25, 65636571.

(29) Samarasinghe, S. D.; Campbell, D. M.; J onas, A.; J onas, J. High-resolution NMR study of the pressure-induced unfolding of lysozyme. Biochemistry 1992, 31, 7773-7778.

(30) Akasaka, K.; Tezuka, T.; Yamada, H. Pressure-induced changes in the folded structure of Iysozyme. J. Mol. Biol. 1997, 271, 671-678.
(31) Urayama, P.; Phillips, G. N..; Gruner, S. M. Probing substates in sperm whale myoglobin using high-pressure crystallography. Struct. Fold. Des. 2002, 10, 51-60.

(32) Kundrot, C. E.; Richards, F. M. Crystal structure of hen egg-white lysozyme at a hydrostatic pressure of 1000 atm. J . Mol. Biol. 1987, 193, 157-170.

(33) Refaee, M.; Tezuka, T.; Akasaka, K.; Williamson, M. P. Pressure-dependent changes in the solution structure of hen egg-white lysozyme. J . Mol. Biol. 2003, 327, 857-865.

(34) Dyson, H. J .; Wright, P. E. Peptide conformation and protein folding. Curr. Opin. Struct. Biol. 1993, 3, 60-67.

(35) Smith, L. J .; Alexandrescu, A. T.; Pitkeathly, M.; Dobson, C. M. Solution structure of a peptide fragment of human $\alpha$-lactalbumin in trifluoroethanol: a model for local structure in the molten globule. Structure 1994, 2, 703-709.

(36) Lavialle, F.; Adams, R. G.; Levin, I. W. Establishment of plasma membrane polarity in mammary epithelial cells correlates with changes in prolactin trafficking and in annexin VI recruitment to membranes. Biochemistry 1982, 21, 2305-2312.

(37) Bazzo, R.; Tappin, M.J .; Pastore, A.; Harvey, T. S.; Carver, J. A.; Campbell, I. D. The structure of melittin. A ${ }^{1} \mathrm{H}$ NMR study in methanol. Eur. J . Biochem. 1988, 173, 139-145.

(38) Thomas, P. D.; Dill, K. A. Local and nonlocal interactions in gl obular proteins and mechanisms of alcohol denaturation. Protein Sci. 1993, 2, 2050-2056.

(39) Liu, Y.; Bolen, D. W. The peptide backbone plays a dominant role in protein stabilization by naturally occurring osmolytes. Biochemistry 1995, 34, 12884-12891.

(40) Liu, H.-L.; Hsu, C.-M. The effects of solvent and temperature on the structural integrity of monomeric melittin by molecular dynamics simulations. Chem. Phys. Lett. 2003, 275, 119-125.

(41) Allen, M. P.; Tildesley, D. J . Computer Simulation of Liquid; Clarendon Press: Oxford, UK, 1987.

(42) McCammon, J . A.; Harvey, S. C. Dynamics of Proteins and Nudeic Acids; Cambridge University Press: London, 1987.

(43) Rapaport, D. C. TheArt of Molecular Dynamics Simulation; Cambridge University Press: Cambridge, UK, 1995.

(44) Liu, H.-L.; Wang, W.-C. The predicted unfolding order of the $\beta$-strands in the starch binding domain from Aspergillus niger glucoamylase. Chem. Phys. Lett. 2002, 366, 284-290.

(45) Liu, H.-L.; Wang, W.-C. Molecular dynamics simulations of the unfol ding of thestarch binding domain from Aspergillus niger glucoamylase. J . Biomol. Struct. Dyn. 2003, 20, 615622.

(46) Liu, H.-L.; Wang, W.-C.; Hsu, C.-M. Molecular dynamics simulations of the unfolding mechanism of the catalytic domain from Aspergillus awamori var. X100 glucoamylase. J . Biomol. Struct. Dyn. 2003, 20, 567-574.

(47) Liu, H.-L.; Ho, Y.; Hsu, C.-M. The influence of metal ions on the substrate binding pocket of the human alcohol dehydrogenase $\beta_{2} \beta_{2}$ by molecular modeling. Chem. Phys. Lett. 2003, 372, 249-254.

(48) Liu, H.-L.; Ho, Y.; Hsu, C.-M. The effect of metal ions on the binding of ethanol to human alcohol dehydrogenase $\beta_{2} \beta_{2}$. J. Biomed. Sci. 2003, 10, 302-312.

(49) Liu, H.-L.; Shu, Y.-C.; Wu, Y.-H. Molecular dynamics simulations to determine the optimal loop length in the helixloop-helix motif. J . Biomol. Struct. Dyn. 2003, 20, 741-746.

(50) Steinrauf, L. K. Structures of monoclinic lysozyme iodide at $1.6 \mathrm{~A}$ and of triclinic lysozyme nitrate at $1.1 \AA$. Acta Crystallogr.c Sect. D: Biol. Crystallogr. 1998, 54, 767-780.

(51) Hwang, M.-J .; Ni, X.; Waldman, M.; E wig, C. S.; Hagler, A. T. Derivation of class II force fields. VI. carbohydrate compounds and anomeric effects. Bi opol ymers 1998, 45, 435468.

(52) Maple, J . R.; Hwang, M.-J .; J alkanen, K. J .; Stockfisch, T. P.; Hagler, A. T. Derivation of class II force fields: V. Quantum force field for amides, peptides, and related compunds. J . Comp. Chem. 1998, 19, 430-458.

(53) Peng, Z.; Ewig, C. S.; Hwang, M.-J .; Waldman, M.; Hagler, A. T. Derivation of class II force fields. 4. van der Waals parameters of alkali metal cations and halide anions. J . Phys. Chem. A 1997, 101, 7243-7252. 
(54) Berendsen, H. J . C.; Postma, J . P. M.; van Gunsteren, W. F.; DiNola, A.; Haak, J . R. Molecular dynamics with coupling to an ecternal bath. J . Comput. Phys. 1984, 81, 3684-3690.

(55) Kazmirski, S. L.; Daggett, V. Simulation of the structural and dynamic properties of unfol ded proteins: the "molten coil" state of bovine pancreatic trypsin inhibitor. J . Mol. Biol. 1998, 284, 793-806.

(56) Kabsch, W. A solution for the best rotation to relate two sets of vectors. Acta Crystallogr., Sect. A: Found. Crystallogr. 1976, 32, 922-923.

(57) Kabsch, W.; Sander, C. Dictionary of protein secondary structure: Pattern recognition of hydrogen-bonded and geometrical features. Biopolymers 1983, 22, 2577-2637.

(58) Heremans, K.; Smeller, L. Protein structure and dynamics at high pressure. Biochim. Biophys. Acta 1998, 1386, 353370.

(59) Kitchen, D. B.; Reed, L. H.; Levy, R. M. Molecular dynamics simulation of solvated protein at high pressure. Biochemistry 1992, 31, 10083-10093.

(60) Wroblowski, B.; Diaz, J . F.; Heremans, K.; Engel borghs, $Y$. Molecular mechanisms of pressure induced conformational changes in BPTI. Proteins Struct. Funct. Genet. 1996, 25, 446-455.

(61) Goossens, K.; Smeller, L.; Frank, J .; Heremans, K. Conformation of bovine pancreatic trypsin inhibitor studied by Fourier transform infrared spectroscopy. Eur. J . Biochem. 1996, 236, 254-262.

(62) Weber, G.; Drickamer, H. G. The effect of high pressure upon proteins and other biomolecules. Quart. Rev. Biophys. 1983, 16, 89-112.
(63) Krimm, S.; Bandekar, J. Vibrational spectroscopy and conformation of peptides, polypeptides and proteins. Adv. Protein Chem. 1986, 38, 181-364.

(64) Ezaki, S.; Hayashi, R. High-pressure effects on starch: structural change and retrogradation, In High Pressure and Biotechnol ogy; Balny, C., Hayashi, R., Heremans, K., Masson, P., Eds.; J ohn Libbey Eurotext: Montrouge, 1992; pp 163165.

(65) Gromiha, M.; Ponnuswamy, P. K. Relationship between amino acid properties and protein compressibility. J . Theor. Biol. 1993, 165, 87-100.

(66) Williams, S.; Causgrove, T. P.; Gilmanshin, R.; Fang, K. S.; Callender, R. H.; Woodruff, W. H.; Dyer, R. B. Fast events in protein folding: helix melting and formation in a small peptide. Biochemistry 1996, 35, 691-697.

(67) Muñoz, V.; Henry, E. R.; Hofrichter, J .; Eaton, W. A. A statistical mechanical model for $\beta$-hairpin kinetics. Proc. Natl. Acad. Sci. U.S.A. 1998, 95, 5872-5879.

(68) Chan, H. S.; Dill, K. A. Origins of structure in globular proteins. Proc. Natl. Acad. Sci. U.S.A. 1990, 87, 6388-6392.

(69) Williams, M. A.; Thornton, J . M. Goodfellow, J . M. Modelling protein unfolding: hen egg-white lysozyme. Protein Eng. 1997, 10, 895-903.

Accepted for publication J anuary 13, 2004.

BP034313K 